\title{
Le rôle des entreprises extérieures et la maintenance des installations du cycle du combustible nucléaire : l'expérience de la Cogéma*
}

\author{
F. CHEVILLARD** \\ (Manuscrit reçu le 3 juin 1985)
}

\begin{abstract}
RÉSUMÉ
L'intervention des entreprises extérieures est analysée dans trois types d'installations du secteur "métallurgie": : séparation isotopique, fabrication des combustibles, retraitement des combustibles irradiés.
\end{abstract}

\section{ABSTRACT}

The operations of contractors are analysed in the metallurgical sector of three types of plants : isotopic separation, fuel fabrication and spent fuel reprocessing.

\section{INTRODUCTION}

La Compagnie générale des matières nucléaires (Cogéma) a été créée en 1976 par la filialisation de la direction des productions du Commissariat à l'énergie atomique. Cette entité du secteur public français, dont le métier est la mise au point et le traitement du combustible nucléaire, est organisée sous la forme d'une société anonyme de droit privé ; elle a réalisé en 1984 un chiffre d'affaires hors taxes d'environ 10380 MFF, dont 4660 MFF pour son secteur "mines" et 5720 MFF pour son secteur "métallurgie". Elle emploie 2661 personnes dans ses activités minières (recherche - extraction - traitement des minerais) et 6608 personnes dans ses activités métallurgiques (séparation isotopique - retraitement). Dans ce domaine, elle contrôle, en outre, directement ou en association, d'autres sociétés dans la conversion, la séparation isotopique et la fabrication des combustibles.

Le présent article est centré sur le secteur "métallurgie" et l'intervention des entreprises extérieures est analysée dans trois types d'installations : séparation isotopique, fabrication des combustibles, retraitement des combustibles irradiés.

* Communication présentée au colloque organisé à Bruxelles du 20 au 22 mars 1985 par les Sociétés belge et française de radioprotection sur la "Radioprotection du personnel des entreprises extérieures intervenant dans les installations nucléaires".

** Compagnie générale des matières nucléaires (Cogéma), BP 4, 78141 VelizyVillacoublay Cedex. 


\section{LE DOMAINE D'INTERVENTION DES ENTREPRISES EXTERIEURES}

\subsection{La politique de sous-traitance}

L'évolution de la technologie, de la capacité et de la complexité des installations rend nécessaire une réflexion approfondie des exploitants à propos de l'intervention des entreprises extérieures. A la maintenance traditionnelle, curative ou préventive, tendent à s'ajouter d'autres types d'interventions, différenciés selon les matériels et les procédés : par exemple, les opérations d'entretien nécessaires sont désormais étudiés dès la conception ; le recours à l'informatique rend possible la saisie, l'exploitation et le suivi des nombreuses données relatives à un matériel ou une pièce. Bien entendu, le niveau de qualification des entreprises et de leur personnel doit s'adapter à ces nouvelles donnes. Quant aux niveaux d'intervention, ils sont aujourd'hui répertoriés pour la pratique par la norme AFNOR [1] qui permet de trouver un langage commun à l'exploitant et aux entreprises (voir annexe).

Une fois définis les types et les niveaux d'intervention, l'exploitant doit avant tout déterminer les travaux qu'il devra assurer directement et ce qu'il peut (en fonction de critères de sécurité, de disponibilité, ou liés au procédé) ou doit (pour des raisons d'économie, de productivité, de flexibilité) confier à des intervenants extérieurs. Le tableau I précise les critères économiques jouant un rôle dans l'intervention des entreprises extérieures. II est clair, notamment, que la sous-traitance ne doit pas diminuer la maîtrise technologique de l'entreprise. En règle générale, à Cogéma, nous nous spécialisons dans l'intervention en temps réel ou légèrement différé et dans l'analyse, l'organisation, et le contrôle des tâches confiées aux intervenants : les travaux prédictifs de remise à hauteur ou curatifs sont organisés par Cogéma et exécutés par les entreprises locales ou régionales, sur le site ou hors du site. $\mathrm{Ce}$ qui relève de la sécurité fait également partie du "domaine réservé". La détermination du périmètre d'intervention des entreprises est également fonction de leurs connaissances du matériel et des interventions, de l'importance de la quantité des matériels à fournir, des à-coups de fabrication... donc des critères propres aux intervenants (savoir-faire, capacité, souplesse d'intervention,....).

\section{TABLEAU I}

Les critères économiques de l'intervention des entreprises extérieures

\begin{tabular}{|ll|}
\hline \multicolumn{2}{|c|}{ POURQUOI RECOURIR AUX ENTREPRISES EXTERIEURES ? } \\
Limiter & les moyens propres affectés aux tâches non direc- \\
& tement productives. \\
Optimiser & les moyens propres \\
& en sous-traitant : \\
& - les pointes ; \\
& - les travaux spécifiques exigeant des moyens dif- \\
& ficiles à amortir. \\
Maîtriser & les coûts des opérations d'entretien. \\
\hline
\end{tabular}




\subsection{Les secteurs d'intervention des entreprises extérieures}

L'inventaire des entreprises intervenantes dans les trois types d'usines précités permet de les classer en deux catégories principales, selon les corps de métiers :

- la logistique générale, comprenant les opérations de nettoyage, de transport, et la restauration ;

- la maintenance nucléaire proprement dite. A des degrés variables, on retrouve dans tous les cas les mêmes sept métiers : mécanique générale et chaudronnerie/tuyauterie, assainissement radioactif, réseaux de distribution d'électricité-fluides, contrôle des systèmes, régulation, automatismes et informatique, blanchisserie nucléaire, transmissions, travaux de bâtiment.

Le tableau II précise le type de travaux relevant de l'assainissement radioactif.

TABLEAU ॥

Travaux relevant de l'assainissement radioactif

- Travaux d'entretien préventifs et programmés.

- Travaux d'entretien correctifs.

- Travaux d'intervention sur incidents radioactifs.

- Travaux de modification de matériels et/ou d'installations.

- Travaux d'élimination d'installations (démantèlement).

Pour une usine de retraitement comme La Hague, les entreprises intervenant en soutien technique peuvent atteindre la cinquantaine dont la moitié assure un courant régulier de travaux en maintenance. On arrive à un effectif total de l'ordre de 300 hommes/an (plus environ 160 pour le soutien logistique). Evidemment, ce nombre va croître avec les extensions et pourraient atteindre, à la fin de la décennie, 650 hommes/an pour la maintenance et 350 pour la logistique générale.

Dans l'enrichissement, on retrouve les mêmes types d'entreprises liées aux interventions (mécanique-électricité...) et les entreprises de reconditionnement de pièces et d'ensembles (garnitures...) pour un total d'environ 30 à 35 entreprises. Ce nombre est nettement inférieur pour une usine de fabrication.

Très souvent, ces entreprises extérieures sont, en fait, des agences de société à vocation nationale qui parfois assurent le service après-vente, connaissant bien le métier, les modalités du travail et les qualifications requises. On assiste, toutefois, à l'émergence de groupes régionaux et d'entreprises locales qui se sont initiés à nos installations et sont opérationnelles.

\section{LES RELATIONS ENTRE L'EXPLOITANT ET LES ENTREPRISES EXTERIEURES}

Les relations doivent faire l'objet d'une analyse si l'on veut bien saisir quels sont les devoirs de ces derniers, notamment en matière de gestion du personnel. 


\subsection{Les modalités contractuelles}

La spécificité et la technicité croissante des tâches confiées aux intervenants impliquent le recours à des modalités contractuelles qui leur imposent une obligation de résultat, mais également rendent nécessaire la disposition d'un soutien technique sur le site ou à proximité immédiate.

Qu'il s'agisse de sous-traitance globale ou de sous-traitance de réalisation, l'exploitant privilégie, de plus en plus, les contrats de résultat, à l'exception des petits travaux urgents, mal définis ou répétitifs qui peuvent, à la limite, faire l'objet de bordereaux ou de dépenses plafonnées.

Dans le contrat de résultat, le prestataire est libre de choisir les moyens à mettre en œuvre et les solutions techniques à imposer. II lui revient donc de s'occuper prioritairement de l'effectif et de sa qualification. Cela l'aide à acquérir une bonne connaissance des lieux, des machines, du savoir-faire et des coûts, mais implique de sa part, un haut niveau de technicité et un personnel qualifié et formé aux modalités d'intervention en milieu nucléaire.

Toutefois, la définition du périmètre et des modalités contractuelles d'intervention des entreprises est insuffisante pour assurer la sécurité des fournitures. En effet, une implantation locale des sous-traitants est très vivement souhaitée. Cogéma est amenée, parfois, à leur apporter un soutien technique.

Bien souvent, les entreprises nationales ayant participé à la construction des usines ont laissé sur place un important service après-vente. Les entreprises locales ont pu également s'initier à la marche des installations. Aux uns comme aux autres, Cogéma apporte des moyens, variables selon la nature des tâches qui leur sont confiées. Ces moyens vont de la mise à disposition sur site d'ateliers, avec leur outillage spécifique, pour certaines entreprises au forfait, à la fourniture de bureaux et de locaux à pièces détachées pour les entreprises titulaires de marchés-cadres, les ateliers de ces dernières étant hors établissement. La standardisation croissante des équipements se prête d'ailleurs à la mise en place d'infrastructures communes à plusieurs ensembles, ce qui renforce la nécessité d'équipes techniques de soutien très compétentes et rompues à ces matériels. Le tableau III récapitule le type de contrats en fonction des tâches à réaliser.

\subsection{L'organisation de la radioprotection}

L'accueil et le suivi du personnel des entreprises intervenantes est le dernier point à envisager dans les relations client-fournisseur : nous considérons, en effet, que le facteur humain est fondamental en matière de maintenance nucléaire. Ce domaine relève de l'application de la loi de 1976 sur l'information et la prévention des accidents du travail, et du respect des dispositions du décret de 1975 relatif à la protection des travailleurs contre les rayonnements ionisants dans les installations nucléaires de base. 
TABLEAU III

Les types de contrats et leurs applications

\begin{tabular}{|c|c|}
\hline \multicolumn{2}{|c|}{ MAINTENANCE } \\
\hline Types de contrats & Applications \\
\hline Forfait & $\begin{array}{l}\text { Travaux importants - préétablis - } \\
\text { spécifiables }\end{array}$ \\
\hline Bordereau & Petits travaux urgents ou répétitifs \\
\hline Dépenses plafonnées & Travaux difficilement spécifiables \\
\hline Forfait partiel (plafonné ou non) & Travaux en partie spécifiables \\
\hline Sur expertise & $\begin{array}{l}\text { Suppose une évaluation du travail } \\
\text { (devis) }\end{array}$ \\
\hline Entretien avec intéressement & Interventions planifiées \\
\hline
\end{tabular}

TABLEAU IV

Radioprotection : Les diverses étapes de l'accueil et du suivi du personnel des entreprises extérieures

- Information sur formalités légales

- Formalités d'accès...

- Adhésion de l'entreprise à l'association de médecine du travail,...

\begin{tabular}{|c|c|}
\hline \multicolumn{3}{|c|}{ Association de médecine du travail } \\
\hline \multicolumn{2}{|c|}{ Visite médicale } \\
de travaux/rayonnements
\end{tabular}

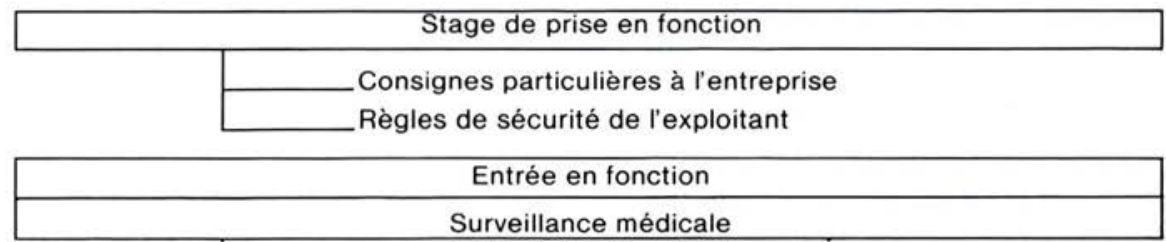

Fourniture des équipements (films - stylos)

Suivi de la dosimétrie

Après l'information de l'entreprise sur les formalités qu'elle doit remplir, pour elle et son personnel, après la définition des zones d'intervention, notamment les travailleurs DATR et NDATR (1), le pivot des procédures est constitué par la visite médicale des personnels : les entreprises adhè-

(1) DATR : directement affectés à des travaux sous rayonnements. NADTR : non directement affectés à des travaux sous rayonnements. 


\section{F. CHEVILLARD}

rent obligatoirement à une association de médecine du travail qui délivre les habilitations de travaux sous rayonnements, soit directement, soit par validation des carnets DATR. L'entrée sur le site commence alors, pour les personnels en cause, par une formation à la sécurité, appelée également "stage de prise de fonction" permettant de faire connaître les consignes particulières à l'entreprise et les règles fondamentales de sécurité de l'exploitant. Enfin, l'exploitant assure la surveillance radiologique des personnels. Le tableau IV schématise les diverses étapes de l'accueil et du suivi du personnel des entreprises extérieures.

\section{CONCLUSION}

L'exploitant nucléaire a donc des responsabilités, d'ailleurs définies par la loi. Cogéma va actuellement très loin. Elle s'est, d'ailleurs, fixée pour objectif de réduire au mieux les doses, et les résultats démontrent que cet objectif est tenu. Les entreprises intervenantes ont également des responsabilités, en particulier pour la formation de leur personnel, non seulement à ses fonctions propres, mais également à la sécurité nucléaire et classique. Nous attendons des entreprises extérieures qu'elles ne viennent pas travailler dans le nucléaire sans s'y être sérieusement préparées, et sans avoir investi dans le domaine de la sécurité : les meilleures le font déjà et auront, nous le souhaitons, un effet d'entraînement sur les autres.

\section{ANNEXE I}

\section{NORME AFNOR X 60.104}

\section{Niveaux de maintenance (extraits)}

\section{$10.11^{\text {er }}$ niveau}

Réglages simples prévus par le constructeur au moyen d'organes accessibles sans aucun démontage ou ouverture de l'équipement ou échanges d'éléments consommables accessibles en toute sécurité tels que voyants ou certains fusibles, etc.

\section{$10.22^{\circ}$ niveau}

Dépannages par échange standard des éléments prévus à cet effet et opérations mineures de maintenance préventive telles que graissage ou contrôle de bon fonctionnement.

\section{$10.33^{\circ}$ niveau}

Identification et diagnostic des pannes, réparations par échange de composants ou d'éléments fonctionnels, réparations mécaniques mineures et toutes réparations courantes de maintenance préventive telles que réglage général ou résignement des appareils de mesure.

$10.44^{e}$ niveau

Tous les travaux importants de maintenance corrective ou préventive à l'exeeption de la rénovation et de la reconstruction. Ce niveau comprend aussi le réglage des appareils de mesure utilisés pour la maintenance et éventuellement la vérification des étalons de travail par les organismes spécialisés.

\section{$10.55^{\circ}$ niveau}

Réparation, reconstruction ou exécution des réparations importantes confiées à un atelier central ou à une unité extérieure. 